\title{
Health-Related Quality Of Life And Its Determinants Among Recreational Drug Users In A Rural Area In Cameroon: A Cross- Sectional Study
}

Atabong Emmanuel Njingu ( $\nabla$ aenjingu@yahoo.com )

University of Buea https://orcid.org/0000-0001-7682-8574

Fombo Enjeh Jabbossung

University of Buea Faculty of Health Sciences

Nyuydzedze Stanley Sunjo

University of Buea Faculty of Health Sciences

Stephen Eyong Njang Ayongi

Ministry of Public Health: Ministere de la Sante Publique

Nembulefac Derick Kemndah

Ministry of Public Health: Ministere de la Sante Publique

Research

Keywords: Recreational drugs, quality of life, WHOQOL-BREF

Posted Date: September 29th, 2021

DOI: https://doi.org/10.21203/rs.3.rs-907905/v1

License: (9) (1) This work is licensed under a Creative Commons Attribution 4.0 International License. Read Full License 


\section{Abstract \\ Background}

This study was aimed to evaluate the health-related quality of life (HRQOL) and its drivers among recreational drug users, compared to non-users using WHOQOL-BREF.

\section{Methods}

A total of 246 recreational drug users and 141 non-recreational drug users were recruited using consecutive sampling of adults in the community. Socio-demographic data, data related to recreational drug use and HRQoL were collected. Data were compared using t-test, analysis of variance and chi-square test. Determinants of HRQoL were obtained using multivariate regression models.

\section{Results}

The mean overall quality of life (OQOL) score was significantly higher for non-recreational drug users compared to users $(75.89 \pm 22.64$ vs $61.08 \pm 23.94$, respectively, $p<0.001)$, non-users as well had significantly higher mean scores across all four domains of WHOQOL-BREF. After multivariable adjustment, use of recreational drugs negatively affected the psychological domain $(\beta=-4.17,95 \% \mathrm{Cl}-8.22$ to $-0,13)$ and the environmental domain $(\beta=-4.47,95 \% \mathrm{Cl}-8.48$ to -0.45$)$. Years of recreational drug use affected the social relationship domain ( $\beta=-0.55$, $95 \% \mathrm{Cl}-0.88$ to -0.22$)$, OQOL ( $\beta=-0.57,95 \% \mathrm{Cl}-0.94$ to -0.19 ), and general health satisfaction ( $\beta=-0.71,95 \% \mathrm{Cl}-1.08$ to -0.35$)$. Number of recreational drugs used contributed to poorer physical health $(\beta=-1.45,95 \% \mathrm{Cl}-2.57$ to -0.33$)$, psychological domain $(\beta=-2.04,95 \% \mathrm{Cl}-3.18$ to -0.91$)$, social relationship domain $(\beta=-1.87,95 \% \mathrm{Cl}-3.39$ to -0.35$)$ as well as overall quality of life $(\beta=-2.13,95 \% \mathrm{Cl}-3.89$ to -0.37$)$. Besides recreational drugs, monthly income also affected physical health ( $\beta=5.17,95 \% \mathrm{Cl} 2.96$ to 7.38$)$, psychological domain $(\beta=3.34$, $95 \% \mathrm{Cl} 1.10$ to 5.58 ), environmental domain ( $\beta=2.64,95 \% \mathrm{Cl} 0.42$ to 4.85$)$ and also the OQOL ( $\beta=4.16,95 \% \mathrm{Cl} 0.70$ to 7.62 ).

\section{Conclusions}

Our findings suggest that, recreational drugs significantly negatively affect the health-related quality of life of its users. People who use multiple recreational drugs and longer years of recreational drug use had a more widespread effect across the health domains. Higher monthly income could improve the HRQoL of recreational drug users.

\section{Background}

Legal and illegal drugs used without medical supervision are referred to as recreational drugs [1]. Alcohol and other substances have for many years been utilized for recreational reasons. Alcohol falls in the category of recreational drugs known as depressants, other categories of recreational drugs includes; analgesics, stimulants and hallucinogens [1,2]. Drug abuse is growing public health problem in both low and high income countries, according world drug report in 2018 , the number of deaths linked to drug abuse increased by $60 \%$ between the year 2000 and 2018 [3-7].

Cameroon is no different from the rest of the world when it comes to drug abuse. A study in 1996 found a diverse use of recreational drugs among males and female of all age groups [8], another study in Cameroon reported the prevalence of recreational drug use to be $1.64 \%$ among medical and nursing students in 2018 [9].

One of the important impacts of recreational drug use on the society is the negative effects on the health of its users. Use of alcohol and other recreational drugs have been identified as risk factors for non-communicable diseases, mental health problems, accidents and high risk behaviours. Drug use also inflicts heavy financial burden on individuals and community [10-13].

According to the World Health Organization (WHO), quality of life (QoL) refers to an individual's perception of his or her position in life, in the context of the culture and value systems in which he or she lives, and in relation to his or her goals, expectations, standards and concerns $[14,15]$. Health-related quality of life (HRQoL) is at times used interchangeably used with QoL, however, some authors consider it to be different or a subset of QoL [16,17]. HRQoL can be defined as an individual's assessment of his/her experiences, and expectations in health-related aspects of his/her life, notably; physical function, psychological well-being, subjective symptoms, social and cognitive 
functions. It is also thought to extend to the person's perception of health correlates like health risks, social support, cultural beliefs and economic status [16-18].

Recreational drug use and the health effects of recreational drug use have been shown to affect the HRQoL of individuals $[19,20]$. In Cameroon, several studies [15,21-24] have been carried out to evaluate the QoL of people living with chronic health conditions. However, no study has been carried out to assess the QoL of recreational drug users in Cameroon. Our study fills this gap by evaluating the HRQoL and factors affecting the HRQoL of recreational drug users compared to non-users of recreational drugs in Cameroon.

\section{Methods}

\section{Study design and setting}

This was a community-based, observational, cross-sectional study. This study was conducted from March 2021 to May 2021 in Mamfe. Mamfe is a border town in the Southwest Region of Cameroon that is about $74 \mathrm{~km}$ away from Nigeria. Mamfe has a population of about 34,225 inhabitants spread over a surface area of 744 square kilometers (population density 46 persons $/ \mathrm{km}^{2}$ ). Mamfe is one of the areas affected by the ongoing socio-political crisis in Cameroon [25,26].

\section{Sampling technique and study participants}

A target sample size of 374 was obtained using a sample size calculator [27]. A margin of error of $\pm 5 \%$, confidence level of $95 \%$ and a $50 \%$ response distribution were used. A population of 13,690 people was used since about $40 \%$ of the 34,225 inhabitants of Mamfe are adults [26], this study had 387 participants.

Consecutive sampling was used to recruit all individuals aged 18 years and above, that are permanent residents of Mamfe or have lived in Mamfe for at least 12 months and consented to the study. We excluded adults who were sick at the time of the study, those with chronic diseases, did not understand English and those with physical or mental disability.

\section{Definition of terms and variables}

Recreational drugs were defined as legal and illegal drugs used without medical supervision [1]. By this definition, alcohol, cigarettes, opioids and other illicit drugs were considered as creational drugs in this study

A recreational drug user was defined as someone who has consumed any recreational drug over the past 12 months [28]. A single question was used to assess this. There is evidence that using a single question for this purpose is valid for screening and evaluation of recreational drugs use [29]. The list of drugs included; alcohol, cigarettes, shisha, cannabis, cocaine, amphetamines, heroin, ecstasy, tramadol, diazepam and sleeping pills, lysergic acid diethylamide (LSD), erection enhancing drugs such as sildenafil. A non-recreational drug user was defined as an individual that has never used drugs for recreational purposes.

Units of alcohol consumed per week was calculated as $5 \%$ x volume of beer (in $\mathrm{ml}$ ) consumed per week/1000 [30]. The average concentration of alcohol in alcoholic beer in Cameroon is $5 \%$.

Physical type of employment generally referred to unskilled jobs such as farming, laborer, and other activities that involves mass lifting of $>20 \mathrm{~kg}$. Non-physical type employment referred to skilled jobs and other employment types with mass lifting of <20 kg [31].

\section{Study procedures and data collection}

Individuals who met the eligibility criteria and consented to the study were interviewed using a pretested structured questionnaire. A doorto-door approach was used to locate participants. To avoid double counting, each individual was asked if they had filled a similar questionnaire prior to recruitment for the study. Literate persons were allowed to fill the questionnaire by themselves after receiving instructions on how to fill the questionnaire. A face-to-face interview was done for illiterate people. Data collected were sociodemographic information, information on the use of recreational drugs and quality of life assessment of participants. Questionnaire was available in English.

The following sociodemographic variables were collected; gender, age, marital status (single, married or widowed), residence (internally displaced, not internally displaced), employment status (employed, housewife, student, unemployed/retired), employment type (physical, non-physical, or combined), level of education (no education, primary, secondary and tertiary education) and average monthly income (< 
FCFA50.000, FCFA50.000-FCFA100.000, FCFA100.000-FCFA300000, and > FCFA300 000 FCFA (US $\$ 1=$ FCFA530)). Information on the use of recreational drugs, types and frequency of drug used were also collected.

The WHOQOL-BREF questionnaire [32] was used to assess the health-related quality of life (HRQoL) of participants. The WHOQOL-BREF tool is a generic self-report HRQoL questionnaire. It was designed to be cross-culturally applicable and has been used in clinical practice and research to assess health outcomes, follow-up progression of a disease and compare health states. Compared to other HRQoL evaluation tools such as Short Form 36, WHOQOL-BREF was found to have good-to-excellent psychometric properties across health states $[33,34]$.

The WHOQOL-BREF questionnaire consists of 26 questions, of which 24 are divided into four domains; physical health domain (PHD), psychological domain (PSD), environmental domain (END) and social relationships domain (SRD). The other two items in the questionnaire evaluates an individual's satisfaction with their state of health (general health score) and individual's self-perceived quality of life (OQoL score) [32]. The WHOQOL-BREF tool was chosen because of its cross-cultural applicability, low administrative burden, sensitivity and availability in English which is the official language of the study population. Each domain consists of several facets and questions are scored in a 5-point Likert response scale. Item scores obtained were transformed into domain scores using the procedure described in WHOQOL-BREF manual [32]. Scores are converted on a scale of 0 to 100 to allow for comparisons to be made across domains with unequal number of items. Scores were organized such that higher scores imply better health-related quality of life [32,35].

\section{Data management and data analysis}

Data collected were entered into Microsoft excel 2016, were cleaned, exported and was analyzed using Statistical Package for Social Sciences (SPSS v20). Continuous variables were summarized using mean and standard deviation. Categorical variables were summarized using counts and percentages. Sociodemographic variables were compared between recreational drug users and non-recreational drug users using Pearson's chi-square or Fisher's exact test. Mean quality of life scores across various domains were compared using independent t-test or one-way ANOVA. We used multivariate linear regression models to determine factors independently associated with WHOQOL-BREF scores. Statistical significance was set at $p<0.05$.

\section{Results}

A total of 246 recreational drug users and 141 non-recreational drug users were recruited for this study. The mean age of participants was 34.54 years $(S D=10.96)$, the male to female ratio was $1.10: 1$, majority of the study population were single $(48.70 \%)$ and employed (55.30\%). Details of the sociodemographic features of recreational drug users and non-recreational drug users are shown in Table 1. Higher proportion of males (64.23\%) compared to females used recreational drugs, people who were single (48.36\%), employed (54.07\%), have average monthly income of $<50,000$ FCFA $(50.0 \%)$, attained secondary education (45.93\%) and those with physical type of employment (48.78\%) had higher proportion of recreational drug use. 
Table 1

; Comparison of sociodemographic characteristics of recreational drug users and non-users

\begin{tabular}{|c|c|c|c|}
\hline Sociodemographic variables & Recreational drug users $(n=246)$ & Non-recreational drug users $(n=141)$ & p-value \\
\hline Age (mean, SD) (years) & $36.17(11.68)$ & $31.70(8.92)$ & 0.000 \\
\hline \multicolumn{4}{|l|}{ Gender $(n, \%)$} \\
\hline Female & $88(35.77)$ & $96(68.09)$ & 0.000 \\
\hline Male & $158(64.23)$ & $45(31.91)$ & \\
\hline \multicolumn{4}{|l|}{ Residence (n, \%) } \\
\hline Not internally displaced & $189(76.83)$ & $113(80.14)$ & 0.529 \\
\hline Internally displaced & $57(23.17)$ & $28(19.86)$ & \\
\hline \multicolumn{4}{|l|}{ Marital status (n, \%) } \\
\hline Single & $118(48.36)$ & $69(49.29)$ & 0.742 \\
\hline Married & $111(45.49)$ & $65(46.43)$ & \\
\hline Widowed & $15(6.15)$ & $6(4.29)$ & \\
\hline \multicolumn{4}{|l|}{ Employment status (n, \%) } \\
\hline Employed & $133(54.07)$ & $81(57.45)$ & 0.009 \\
\hline Unemployed & $86(34.96)$ & $40(28.37)$ & \\
\hline Student & $17(6.91)$ & $10(7.09)$ & \\
\hline Housewife & $1(0.41)$ & $8(5.67)$ & \\
\hline Retired & $9(3.66)$ & $2(1.42)$ & \\
\hline \multicolumn{4}{|l|}{ Monthly income (FCFA) (n, \%) } \\
\hline$<50000$ & $123(50.00)$ & $80(56.74)$ & 0.516 \\
\hline $50000-99000$ & $73(29.67)$ & $39(27.66)$ & \\
\hline $100000-299000$ & $43(17.48)$ & $20(14.18)$ & \\
\hline$\geq 300000$ & $7(2.85)$ & $2(1.42)$ & \\
\hline \multicolumn{4}{|l|}{ Level of education (n, \%) } \\
\hline Primary/no formal education & $38(15.45)$ & $18(12.77)$ & 0.583 \\
\hline Secondary & $113(45.93)$ & $72(51.06)$ & \\
\hline Tertiary & $95(38.62)$ & $51(36.17)$ & \\
\hline \multicolumn{4}{|l|}{ Employment type (n, \%) } \\
\hline Non-physical & $85(34.55)$ & $50(35.46)$ & 0.584 \\
\hline Physical & $120(48.78)$ & $73(51.77)$ & \\
\hline Combined & $41(16.67)$ & $18(12.77)$ & \\
\hline
\end{tabular}

Table 2 shows WHOQOL-BREF mean domain score differences between recreational drug users and non-users. Compared to recreational drug users, non-recreational drug users had significantly better scores across all four domains, as well as significantly better overall quality of life (OQOL) and general health satisfaction (GHS). Among recreational drug users, the highest score was in the psychological domain while the environmental and social relationship domains had the lowest scores. 
Table 2

; WHO-BREF domain score differences between recreational and non-recreational drug users

\begin{tabular}{|lll|}
\hline WHO-BREF domain & Recreational drug users mean (SD) & Non-recreational drug users mean (SD) \\
\hline Physical health & $59.28(15.37)$ & $66.37(12.68)$ \\
\hline Psychological & $61.28(16.24)$ & $71.82(9.72)$ \\
\hline Social relationships & $48.83(19.59)$ & $57.75(18.01)$ \\
\hline Environmental & $46.54(14.51)$ & $52.16(13.86)$ \\
\hline Overall quality of life & $61.08(23.94)$ & $75.89(22.64)$ \\
\hline General health satisfaction & $57.52(23.13)$ & $72.87(19.71)$ \\
\hline $\begin{array}{l}\text { Values are represented in mean and standard deviation (SD). Independent sample t-test was used for comparison, p-value < } 0.05 \\
\text { indicated statistically significant difference }\end{array}$ \\
\hline
\end{tabular}

Variations in the mean WHOQOL-BREF domain scores as well as the OQOL and GHS across sociodemographic variables are shown in Table 3. When compared to males, females had significantly better quality of life (QOL) across all domains, the OQOL and GHS scores were also significantly higher for females. People who are not internally displaced had better scores in the environmental domain (END) and OQOL compared to internal displaced persons $(p<0.05)$. People who are widowed scored higher in the END and social relationship domain (SRD) compared to single and married people ( $p$ 0.005). In terms of employment status, no single demographic characteristic had consistently better scores across all domains, however, retired individuals had significantly worse OQOL and GHS. People with average monthly income $\geq 300,000$ FCFA had superior scores across all aspects of WHOQOL-BREF compared to people with lower monthly income, this superiority was statistically significant in all aspects except in psychological domain (PSD) and SRD. People who have attained tertiary education and people who have used recreational drugs for less than 5 years had significantly better scores across most aspects of WHOQOL-BREF. Compared to their counterparts, people using 2 or more recreational drugs and people who consumed more than 14 units of alcohol per week had significantly poor scores across most aspects of WHOQOL-BREF. 
Table 3

; Association of sociodemographic variables with WHOQOL-BREF domains

\begin{tabular}{|c|c|c|c|c|c|c|}
\hline Variable & PHD & PSD & SRD & END & OQOL & GHS \\
\hline \multicolumn{7}{|l|}{ Gender } \\
\hline Male & $60.27(16.19)$ & $63.35(16.34)$ & $48.55(20.91)$ & $46.93(15.04)$ & $62.07(26.95)$ & $59.61(25.49)$ \\
\hline Female & $63.63(12.98)$ & $67.08(13.34)$ & $55.98(17.00)$ & $50.42(13.71)$ & $71.33(20.50)$ & $66.98(19.57)$ \\
\hline$p$-value & 0.026 & 0.015 & 0.000 & 0.018 & 0.000 & 0.002 \\
\hline \multicolumn{7}{|l|}{ Residence } \\
\hline Not internally displaced & $62.10(14.98)$ & $65.60(14.62)$ & $51.71(18.43)$ & $50.83(13.08)$ & $70.70(21.23)$ & $63.99(21.78)$ \\
\hline Internally displaced & $61.04(14.32)$ & $63.42(16.61)$ & $53.40(22.91)$ & $40.61(16.51)$ & $51.47(29.21)$ & $60.00(27.33)$ \\
\hline$p$-value & 0.561 & 0.240 & 0.481 & 0.000 & 0.000 & 0.160 \\
\hline \multicolumn{7}{|l|}{ Marital status } \\
\hline Single & $63.48(14.72)$ & $64.89(15.61)$ & $50.52(20.29)$ & $46.11(15.62)$ & $65.24(25.70)$ & $62.83(23.67)$ \\
\hline Married & $60.97(14.41)$ & 65.48 (15.09) & $52.49(18.67)$ & $50.63(13.19)$ & $68.75(22.91)$ & $64.63(22.89)$ \\
\hline Widowed & $56.38(18.15)$ & $66.14(10.17)$ & $64.62(14.80)$ & $55.14(10.08)$ & $65.48(20.12)$ & $55.95(19.21)$ \\
\hline$p$-value & 0.058 & 0.896 & 0.007 & 0.001 & 0.372 & 0.250 \\
\hline \multicolumn{7}{|l|}{ Employment status } \\
\hline Employed & $65.72(13.45)$ & $66.68(14.68)$ & $51.98(20.34)$ & $51.61(12.37)$ & $71.38(23.01)$ & $67.76(21.81)$ \\
\hline Unemployed & $56.98(14.23)$ & $60.90(15.80)$ & $51.25(18.65)$ & $44.30(17.09)$ & $60.12(26.11)$ & $55.36(23.58)$ \\
\hline Student & $59.78(19.28)$ & $70.89(13.89)$ & $57.37(16.68)$ & $45.26(14.80)$ & $66.67(20.80)$ & $73.15(18.25)$ \\
\hline Housewife & $62.00(15.61)$ & $72.89(7.62)$ & $47.33(10.01)$ & $44.67(7.62)$ & $58.33(12.50)$ & $58.33(12.50)$ \\
\hline Retired & $47.73(9.63)$ & $62.82(9.68)$ & $54.55(24.07)$ & $50.27(11.35)$ & $50.00(27.39)$ & $40.91(23.11)$ \\
\hline$p$-value & 0.000 & 0.000 & 0.571 & 0.000 & 0.000 & 0.000 \\
\hline \multicolumn{7}{|l|}{ Monthly income (FCFA) } \\
\hline$<50,000$ & $58.85(15.14)$ & $63.74(15.29)$ & $53.17(19.50)$ & $45.77(15.30)$ & $62.32(26.25)$ & $60.47(23.69)$ \\
\hline $50-99000$ & $64.62(14.69)$ & $65.24(15.73)$ & $52.28(20.58)$ & $50.29(13.49)$ & $68.53(20.89)$ & $66.96(21.28)$ \\
\hline $100-299000$ & $65.06(11.48)$ & $68.06(12.48)$ & $48.03(15.98)$ & $53.51(12.08)$ & $73.81(23.08)$ & $62.30(22.84)$ \\
\hline$\geq 300000$ & $73.11(15.81)$ & $74.22(14.85)$ & $53.44(26.27)$ & $56.44(9.50)$ & $83.33(12.50)$ & $80.56(24.30)$ \\
\hline$p$-value & 0.000 & 0.060 & 0.343 & 0.000 & 0.000 & 0.012 \\
\hline \multicolumn{7}{|l|}{ Level of education } \\
\hline Primary/no formal education & $55.91(15.31)$ & $60.89(14.98)$ & $52.68(21.37)$ & $47.82(12.45)$ & $61.61(26.53)$ & $53.13(20.26)$ \\
\hline Secondary & $61.92(15.09)$ & $65.10(15.57)$ & $51.86(18.68)$ & $46.83(16.79)$ & $63.65(25.66)$ & $62.84(23.47)$ \\
\hline Tertiary & $64.07(13.75)$ & $66.78(14.25)$ & $52.14(19.86)$ & $51.12(11.56)$ & $71.92(21.15)$ & $67.29(22.66)$ \\
\hline$p$-value & 0.002 & 0.045 & 0.962 & 0.025 & 0.003 & 0.000 \\
\hline \multicolumn{7}{|l|}{ Employment type } \\
\hline Non-physical & $61.28(15.89)$ & $64.50(15.52)$ & $53.45(19.17)$ & $49.76(14.95)$ & $70.19(21.27)$ & $67.78(23.03)$ \\
\hline Physical & $61.92(14.66)$ & $65.52(15.39)$ & $49.94(18.75)$ & $47.63(14.57)$ & $63.73(26.75)$ & $59.20(23.17)$ \\
\hline Combined & $63.02(12.90)$ & $65.25(13.12)$ & $55.95(21.88)$ & $49.03(13.26)$ & $66.95(22.95)$ & $65.25(21.28)$ \\
\hline
\end{tabular}

Proportions were compared using independent sample t-test and one-way ANOVA. $p<0.005$ indicated significant difference. 


\begin{tabular}{|c|c|c|c|c|c|c|}
\hline Variable & PHD & PSD & SRD & END & OQOL & GHS \\
\hline$p$-value & 0.754 & 0.830 & 0.070 & 0.412 & 0.062 & 0.003 \\
\hline \multicolumn{7}{|c|}{ Years of drug use } \\
\hline$<5$ & $65.63(14.19)$ & $69.15(12.86)$ & $56.85(18.15)$ & $49.44(14.48)$ & 71.35 (24.94) & $70.81(20.51)$ \\
\hline $5-9$ & $59.09(16.62)$ & 61.33 (17.18) & $46.14(17.64)$ & $43.45(15.51)$ & $63.46(20.42)$ & 61.54 (21.95) \\
\hline$\geq 10$ & $57.98(13.21)$ & $61.51(15.34)$ & $48.70(20.86)$ & $50.56(13.23)$ & $61.09(24.96)$ & 52.62 (23.39) \\
\hline$p$-value & 0.000 & 0.000 & 0.000 & 0.002 & 0.001 & 0.000 \\
\hline \multicolumn{7}{|c|}{ Number of drug use } \\
\hline 0 & $66.34(12.63)$ & 73.06 (8.30) & $58.41(17.59)$ & $52.08(13.54)$ & $78.23(20.90)$ & 74.80 (18.72) \\
\hline 1 & $60.12(16.81)$ & $56.98(16.50)$ & $44.83(24.43)$ & $43.95(15.93)$ & $62.08(23.69)$ & $52.03(24.48)$ \\
\hline$\geq 2$ & $55.48(12.57)$ & $57.58(17.510$ & $42.75(18.29)$ & $45.91(14.58)$ & $52.47(22.57)$ & $53.09(21.79)$ \\
\hline$p$-value & 0.000 & 0.000 & 0.000 & 0.000 & 0.000 & 0.000 \\
\hline \multicolumn{7}{|c|}{ Units of alcohol/week } \\
\hline$\leq 14$ & $64.96(14.72)$ & 69.35 (11.92) & 57.03 (19.01) & $51.10(13.73)$ & $72.22(22.83)$ & 69.91 (19.52) \\
\hline$>14$ & $57.99(14.34)$ & $60.06(17.04)$ & $45.94(18.38)$ & $45.55(14.08)$ & $59.26(25.06)$ & $53.86(24.85)$ \\
\hline$p$-value & 0.001 & 0.000 & 0.000 & 0.000 & 0.000 & 0.000 \\
\hline
\end{tabular}

Table 4 shows the results of multivariate linear regression analysis of factors independently associated with WHOQOL-BREF scores. After adjustments for possible confounding factors, increasing age was associated with better $\operatorname{SRD}(\beta=0.39, \mathrm{p}<0.05)$ and poorer GHS $(\beta=$ $-0.34, p<0.05)$. Using recreational drugs affected the PSD $(\beta=-4.17, p<0.05)$ and END $(\beta=-4.47, p<0.05)$. The SRD $(\beta=-0.55, p<0.01)$, OQOL $(\beta=-0.57, p<0.01)$ and GHS $(\beta=-0.71, p<0.001)$ were significantly influenced by years of recreational drug use. The use of multiple recreational drugs influenced the PHD $(\beta=-1.45, p<0.05)$, PSD $(\beta=-2.04, p<0.001)$, SRD $(\beta=-1.87, p<0.05)$ and the 0QOL $(\beta=-2.13, p<$ $0.05)$. Higher pack years of smoking was associated with poorer PSD $(\beta=-0.32, p<0.05), \operatorname{SRD}(\beta=-0.61, p<0.01)$ and $0 Q 0 L(\beta=-0.67, p$ $<0.01)$. Higher monthly income was associated with significantly better $\operatorname{PHD}(\beta=5.17, p<0.001), P S D(\beta=3.34, p<0.01), E N D(\beta=2.64, p$ $<0.05)$ and OQOL $(\beta=4.16, \mathrm{p}<0.05)$. 
Table 4

; Multivariate regression model showing factors independently associated with WHOQOL-BREF scores.

\begin{tabular}{|c|c|c|c|c|c|c|c|c|c|c|c|c|}
\hline \multirow[t]{2}{*}{ Variable } & \multicolumn{2}{|c|}{$\begin{array}{l}\text { PHD } \\
a R^{2}=0.193\end{array}$} & \multicolumn{2}{|c|}{$\begin{array}{l}\text { PSD } \\
a^{2}=0.199\end{array}$} & \multicolumn{2}{|c|}{$\begin{array}{l}\text { SRD } \\
a R^{2}=0.139\end{array}$} & \multicolumn{2}{|c|}{$\begin{array}{l}\text { END } \\
a^{2}=0.150\end{array}$} & \multicolumn{2}{|c|}{$\begin{array}{l}\text { OQOL } \\
\mathrm{aR}^{2}=0.274\end{array}$} & \multicolumn{2}{|c|}{$\begin{array}{l}\text { GHS } \\
a^{2}=0.230\end{array}$} \\
\hline & $\beta$ & $\begin{array}{l}95 \% \\
\mathrm{Cl}\end{array}$ & $\beta$ & $\begin{array}{l}95 \% \\
\mathrm{Cl}\end{array}$ & $\beta$ & $\begin{array}{l}95 \% \\
\mathrm{Cl}\end{array}$ & $\beta$ & $\begin{array}{l}95 \% \\
\mathrm{Cl}\end{array}$ & $\beta$ & $\begin{array}{l}95 \% \\
\mathrm{Cl}\end{array}$ & $\beta$ & $\begin{array}{l}95 \% \\
\mathrm{Cl}\end{array}$ \\
\hline Age in years & -0.21 & $\begin{array}{l}-0.43 \\
0.01\end{array}$ & 0.02 & $\begin{array}{l}-0.20 \\
0.25\end{array}$ & $0.39 *$ & $\begin{array}{l}0.08 \\
0.69\end{array}$ & 0.07 & $\begin{array}{l}-0.16 \\
0.29\end{array}$ & 0.23 & $\begin{array}{l}-0.12 \\
0.58\end{array}$ & $-0.34 *$ & $\begin{array}{l}-0.68 \\
0.00\end{array}$ \\
\hline Gender & -0.83 & $\begin{array}{l}-4.05 \\
2.39\end{array}$ & 2.57 & $\begin{array}{l}-0.70 \\
5.83\end{array}$ & -0.96 & $\begin{array}{l}-5.33 \\
3.41\end{array}$ & -1.27 & $\begin{array}{l}-4.50 \\
1.97\end{array}$ & -1.18 & $\begin{array}{l}-6.23 \\
3.87\end{array}$ & 0.65 & $\begin{array}{l}-4.26 \\
5.55\end{array}$ \\
\hline Marital status & -0.50 & $\begin{array}{l}-3.75 \\
2.75\end{array}$ & 0.04 & $\begin{array}{l}-3.26 \\
3.33\end{array}$ & 2.81 & $\begin{array}{l}-1.61 \\
7.22\end{array}$ & 1.98 & $\begin{array}{l}-1.29 \\
5.24\end{array}$ & -1.01 & $\begin{array}{l}-6.10 \\
4.08\end{array}$ & $6.12^{*}$ & $\begin{array}{l}1.17 \\
11.07\end{array}$ \\
\hline $\begin{array}{l}\text { Level of } \\
\text { education }\end{array}$ & -1.66 & $\begin{array}{l}-4.16 \\
0.84\end{array}$ & 0.12 & $\begin{array}{l}-2.42 \\
2.65\end{array}$ & 0.07 & $\begin{array}{l}-3.32 \\
3.46\end{array}$ & -0.62 & $\begin{array}{l}-3.13 \\
1.89\end{array}$ & -0.16 & $\begin{array}{l}-4.08 \\
3.76\end{array}$ & 1.44 & $\begin{array}{l}-2.37 \\
5.25\end{array}$ \\
\hline $\begin{array}{l}\text { Employment } \\
\text { type }\end{array}$ & 1.50 & $\begin{array}{l}-0.56 \\
3.56\end{array}$ & 1.68 & $\begin{array}{l}-0.40 \\
3.77\end{array}$ & 0.84 & $\begin{array}{l}-1.95 \\
3.64\end{array}$ & 0.12 & $\begin{array}{l}-1.95 \\
2.18\end{array}$ & -1.29 & $\begin{array}{l}-4.52 \\
1.93\end{array}$ & -2.11 & $\begin{array}{l}-5.25 \\
1.02\end{array}$ \\
\hline $\begin{array}{l}\text { Use of } \\
\text { recreational } \\
\text { drugs }\end{array}$ & -1.20 & $\begin{array}{l}-5.20 \\
2.80\end{array}$ & $-4.17 *$ & $\begin{array}{l}-8.22 \\
-0.13\end{array}$ & -0.27 & $\begin{array}{l}-5.70 \\
5.15\end{array}$ & $-4.47 *$ & $\begin{array}{l}-8.48 \\
-0.45\end{array}$ & -3.94 & $\begin{array}{l}-10.20 \\
2.32\end{array}$ & -4.74 & $\begin{array}{l}-10.83, \\
1.35\end{array}$ \\
\hline $\begin{array}{l}\text { Years of } \\
\text { recreational } \\
\text { drug use }\end{array}$ & -0.12 & $\begin{array}{l}-0.36 \\
0.12\end{array}$ & -0.09 & $\begin{array}{l}-0.34 \\
0.15\end{array}$ & $-0.55^{\star \star}$ & $\begin{array}{l}-0.88 \\
-0.22\end{array}$ & 0.07 & $\begin{array}{l}-0.17 \\
0.31\end{array}$ & $-0.57 * *$ & $\begin{array}{l}-0.94 \\
-0.19\end{array}$ & $-0.71 * \star \star$ & $\begin{array}{l}-1.08 \\
-0.35\end{array}$ \\
\hline $\begin{array}{l}\text { Number of } \\
\text { drug use }\end{array}$ & $-1.45^{\star}$ & $\begin{array}{l}-2.57 \\
-0.33\end{array}$ & $-2.04 * \star \star$ & $\begin{array}{l}-3.18 \\
-0.91\end{array}$ & $-1.87^{\star}$ & $\begin{array}{l}-3.39 \\
-0.35\end{array}$ & -0.02 & $\begin{array}{l}-1.15 \\
1.10\end{array}$ & $-2.13^{*}$ & $\begin{array}{l}-3.89 \\
-0.37\end{array}$ & -1.28 & $\begin{array}{l}-2.99 \\
0.43\end{array}$ \\
\hline $\begin{array}{l}\text { Units of } \\
\text { alcohol per } \\
\text { week }\end{array}$ & -0.05 & $\begin{array}{l}-0.14 \\
0.05\end{array}$ & -0.08 & $\begin{array}{l}-0.17 \\
0.02\end{array}$ & 0.05 & $\begin{array}{l}-0.08 \\
0.18\end{array}$ & -0.056 & $\begin{array}{l}-0.15 \\
0.04\end{array}$ & 0.03 & $\begin{array}{l}-0.12 \\
0.18\end{array}$ & 0.03 & $\begin{array}{l}-0.11 \\
0.18\end{array}$ \\
\hline $\begin{array}{l}\text { Pack years of } \\
\text { smoking }\end{array}$ & -0.02 & $\begin{array}{l}-0.33 \\
0.29\end{array}$ & $-0.32^{*}$ & $\begin{array}{l}-0.63 \\
0.00\end{array}$ & -0.61 ** & $\begin{array}{l}-1.03 \\
-0.19\end{array}$ & -0.24 & $\begin{array}{l}-0.55 \\
0.08\end{array}$ & $-0.67 * *$ & $\begin{array}{l}-1.16 \\
-0.18\end{array}$ & -0.18 & $\begin{array}{l}-0.66 \\
0.30\end{array}$ \\
\hline Residence & 1.82 & $\begin{array}{l}-1.66 \\
5.29\end{array}$ & -0.55 & $\begin{array}{l}-4.08 \\
2.97\end{array}$ & 1.78 & $\begin{array}{l}-2.93 \\
6.50\end{array}$ & $-7.32 *$ & $\begin{array}{l}-10.81 \\
-3.83\end{array}$ & -6.70 & $\begin{array}{l}-2.15 \\
-1.25\end{array}$ & -1.67 & $\begin{array}{l}-6.97 \\
3.63\end{array}$ \\
\hline $\begin{array}{l}\text { Employment } \\
\text { status }\end{array}$ & -2.13 & $\begin{array}{l}-3.88 \\
-0.39\end{array}$ & 1.49 & $\begin{array}{l}-0.28 \\
3.26\end{array}$ & -0.89 & $\begin{array}{l}-3.26 \\
1.48\end{array}$ & -1.27 & $\begin{array}{l}-3.03 \\
0.48\end{array}$ & $-2.93^{\star}$ & $\begin{array}{l}5.67 \\
-0.19\end{array}$ & -2.46 & $\begin{array}{l}-5.12 \\
0.198\end{array}$ \\
\hline $\begin{array}{l}\text { Monthly } \\
\text { income(FCFA) }\end{array}$ & $5.17 * \star \star$ & $\begin{array}{l}2.96 \\
7.38\end{array}$ & $3.34^{\star \star}$ & $\begin{array}{l}1.10 \\
5.58\end{array}$ & -1.51 & $\begin{array}{l}0.32 \\
1.48\end{array}$ & $2.64^{*}$ & $\begin{array}{l}0.42 \\
4.85\end{array}$ & $4.16 *$ & $\begin{array}{l}0.70 \\
7.62\end{array}$ & 3.28 & $\begin{array}{l}-0.08 \\
6.65\end{array}$ \\
\hline \multicolumn{13}{|c|}{$\beta=$ beta coefficient, $\mathrm{Cl}=$ confidence interval } \\
\hline \multicolumn{13}{|c|}{$\star \star \star$ Beta coefficient significant at $<0.001$} \\
\hline \multicolumn{13}{|c|}{ ** Beta coefficient significant at $<0.01$} \\
\hline * Beta coeffic & ianifin & $t<0$ & & & & & & & & & & \\
\hline
\end{tabular}

\section{Discussion}

Recreational drug use is a recognized cause of diminished quality of life, the extent of its effect on QOL varies with certain demographic factors such as age, sex and even the type, number and duration of drug use. The aim of this study was to evaluate the HRQoL and its determinants among recreational drug users compared to non-recreational drug users. We found that non-recreational drug users had significantly better scores across all four WHOQOL-BREF domains, as well as significantly better OQOL and GHS when compared to recreational drug users. Other factors such as number and years of recreational drug use, age, gender, marital status and average monthly income also affected the WHOQOL-BREF domain scores. After adjustments, increasing age predicted better PHD but poorer GHS, use of recreational drug was associated with poorer PSD and END. As the years of recreational drug use increased the SRD, OQOL and GHS worsened. Using multiple recreational drugs predicted poorer PHD, PSD, SRD and OQOL. Higher pack years of smoking was associated 
with poorer PSD, SRD and OQOL. Being internally displaced predicted poorer END. And higher monthly income positively influenced PHD, PSD, END and OQOL.

Our study demonstrated that non-recreational drug users have significantly better mean scores across WHOQOL-BREF domains compared to non-users, this is in line with other studies [36-38] carried out in western countries. Unlike other studies [36-38] in which the psychological domain was the most impaired among recreational drug users, our study showed that the environmental domain of the WHOQOL-BREF was the most impaired amongst drug users. This could be due to the fact that this study was carried out in an area with an ongoing civil war, people living in war zones will likely assess their living environment to be unsafe and unhealthy [39]. Even among nonrecreational drug users in this study, the environmental domain had the least score.

The study by Domingo-Salvany et al [40] as well as other western studies [15,34-36] clearly demonstrated that QOL of drug users is made worse by consumption of multiple recreational drugs, longer years of recreational drug use, older age, low average monthly income and low level of education, our findings in this study are similar to what was reported by these studies. The lower QOL of drug users compared to non-users cannot only be explained by duration, number or amount of drugs use but also by the negative effects of drug use in several areas of their lives with varying severity [38].

In this study, females had superior mean WHOQOL-BREF domain scores when compared to men. This differs from other studies $[36,40]$ where females were reported to have poorer QOL. The difference could be due to the fact that far lower proportion (less than half) of women in this study used recreational drugs compared to the other studies and most (over 75\%) of the men in this study were drug users. Hence there were fewer females with the effect of recreational drug use on their health.

After controlling for other factors, number of recreational drugs used was the only factor independently associated with worse physical health score, while monthly income independently predicted better PHD, monthly income was also associated with better PSD, END and OQOL after controlling for other factors. This is similar to findings by Moreira et al in Brazil. According to this study, people with low monthly income were more likely to have poor QOL independent of other factors such as use of recreational drugs [36].

Use of recreational drugs, number of recreational drugs use, years of recreational drug use and pack years of smoking each independently predicted poorer PSD and/or SRD. This finding is similar to other studies in Africa and western countries [15,36-38]. Drug users have been shown to have high prevalence of psychiatric disorders which independently influence their psychological and overall QOL [38].

In this study, apart from average monthly income which positively influence the END, use of recreational drugs and whether an individual is internally displaced or not each independently predicted poor environmental health after controlling for other factors. In another study [36], use of recreational did not affect the END scores. Probable loss of perception of problems related to the environment was suggested as the reason for high END scores among users. In our study being internally displaced and the ongoing sociopolitical crisis in the setting of this study heavily impaired the environmental health of participants.

Our study had some limitations; we used a cross-sectional design which impaired our ability to determine causality which would have been possible with a prospective cohort design. Our findings should be generalized with caution as they likely reflect the situation of a rural area that is affected by civil war. In addition, we did not explicitly assess if there were other ongoing events in the lives of participants that could influenced their responses other than use of recreational drugs. Furthermore, all measures of the WHOQOL-BREF were based on selfreports, the veracity of the responses of individuals cannot be controlled. Finally, there are no population modalities for the use of WHOBREF in Cameroon. The lack of reference for Cameroon limited our ability to precisely analyse health outcomes.

We however reduced some of the limitations, by choosing a widely validated tool that was developed to be applied across different cultures and allows for comparisons throughout various settings. Future studies to develop a tool that is adapted to assess HRQoL for our environment could go a long way to improve the evaluation of the impact of recreational drug use on the HRQoL of individuals in this environment.

\section{Conclusions}

Our findings suggest that, recreational drugs impedes the HRQoL of its users. Number of recreational drugs used, years of drug use, pack years of smoking, age and average monthly income each independently influenced the HRQoL of participants. To the best of our knowledge, this study is the first of its kind in Cameroon to provide evidence of diminished HRQoL among recreational drug users in Cameroon. This could serve as basis for appropriate health promotion and sensitization programs to curb the use of recreational drugs which are proven to be detrimental to the health of its users. 


\section{Abbreviations}

QOL; Quality of life

HRQoL; Health-related quality of life

WHO; World Health Organization

WHOQOL-BREF; World Health Organization Quality of Life Brief

OQOL; Overall quality of life

GHS; General health satisfaction

SPSS; Statistical Package for Social Sciences

Cl; Confidence interval

SD; Standard deviation

\section{Declarations}

\section{Acknowledgements}

We are grateful to all the health personnel in Mamfe who assisted us in collecting data in the community. We equally thankful to all those who took part in this study.

\section{Author's contributions}

AEN: conception and design of the study, data collection, data interpretation and data analysis, drafting and review of the manuscript. FEJ: conception and design of the study, data collection, review of the manuscript. AEN and NSS data analysis, data interpretation, drafting and review of the manuscript. NDK and SENA data interpretation, SENA drafting and review of the manuscript. All authors revised and approved the final version of the manuscript.

\section{Funding}

This work did not receive any funding.

\section{Availability of data materials}

The dataset is available from the corresponding author on reasonable request.

\section{Ethics approval and consent to participate}

Ethical approval was obtained from the Institutional Review Board of the Faculty of Health Sciences of the University of Buea. Administrative approvals were obtained from the Southwest Regional Delegation of Public Health and the District Medical Officer for Mamfe. Participants also gave written consent to willingly participate in this study after careful explanation of the study scope and purpose. Data were kept completely anonymous and confidentiality was maintained during processing of questionnaires.

\section{Consent for publication}

Not applicable

\section{Competing interests}


The authors declare that they have no competing interest.

\section{Publisher's note}

Springer Nature remains neutral with regard to jurisdictional claims in published maps and institutional affiliations.

\section{References}

1. Hawkes N. Sixty seconds on...psilocybin. BMJ. 2016;353:i2775. 2021 Jul 29;i2775.

2. Degenhardt L, Charlson F, Ferrari A, Santomauro D, Erskine H, Mantilla-Herrara A, et al. The global burden of disease attributable to alcohol and drug use in 195 countries and territories, 1990-2016: a systematic analysis for the Global Burden of Disease Study 2016. Lancet Psychiatry. 2018 Dec 1;5(12):987-1012.

3. Watt MH, Meade CS, Kimani S, MacFarlane JC, Choi KW, Skinner D, et al. The impact of methamphetamine ("tik") on a peri-urban community in Cape Town, South Africa. International Journal of Drug Policy. 2014; 25(2):219-25.

https://doi.org/10.1016/j.drugpo.2013.10.007.

4. Abdeta T, Tolessa D, Adorjan K, Abera M. Prevalence, withdrawal symptoms and associated factors of khat chewing among students at Jimma University in Ethiopia. BMC psychiatry. 2017; 17(1):142. https://doi.org/10.1186/s12888-017-1284-4.

5. Abio A, Sezirahiga J, Davis LE, Wilson ML. Substance use and sociodemographic correlates among adolescents in a low-income sub Saharan setting. Journal of Injury and Violence Research. 2020; 12 (1):21. https://doi.org/10.5249/jivr.v12i1.1195.

6. World Drug Report 2018 [Internet]. [cited 2021 Aug 2]. Available from: https://www.unodc.org/wdr2018/

7. Ajayi Al, Somefun OD. Recreational drug use among Nigerian university students: Prevalence, correlates and frequency of use. PloS One. 2020;15(5):e0232964.

8. Wansi E, Sam-Abbenyi A, Befdi-Mengue R, Enyme FN, Ntone FN. Rapid assessment of drug abuse in Cameroon. Bull Narc. $1996 ; 48(1-2): 79-88$.

9. Mbanga CM, Efie DT, Aroke D, Njim T. Prevalence and predictors of recreational drug use among medical and nursing students in Cameroon: a cross sectional analysis. BMC Res Notes. 2018 Jul 28;11(1):515.

10. Substance Abuse. WHO | Regional Office for Africa. [cited 2021 Aug 2]. Available from: https://www.afro.who.int/healthtopics/substance-abuse

11. Volkow ND, Baler RD, Compton WM, Weiss SRB. Adverse health effects of marijuana use. New England Journal of Medicine. 2014; 370(23):2219-27. https://doi.org/10.1056/NEJMra1402309.

12. Di Forti M, Sallis H, Allegri F, Trotta A, Ferraro L, Stilo SA, et al. Daily use, especially of high-potency cannabis, drives the earlier onset of psychosis in cannabis users. Schizophrenia bulletin. 2013; 40 (6):1509-17. https://doi.org/10.1093/schbul/sbt181.

13. Degenhardt L, Hall W. Extent of illicit drug use and dependence, and their contribution to the global burden of disease. The Lancet. 2012; 379(9810):55-70.

14. Group W Study protocol for the world Health organization project to develop a quality of life assessment instrument (WHOQOL). Qual Life Res 1993;2:153-9. 10.1007/BF00435734.

15. Aminde JA, Aminde LN, Bija MD, Lekpa FK, Kwedi FM, Yenshu EV, et al. Health-related quality of life and its determinants in patients with chronic low back pain at a tertiary hospital in Cameroon: a cross-sectional study. BMJ Open [Internet]. 2020 Oct 6 [cited 2021 Jan 23];10(10). Available from: https://www.ncbi.nlm.nih.gov/pmc/articles/PMC7539580/

16. Németh G. Health related quality of life outcome instruments. Eur Spine J 2006;15:S44-51. 10.1007/s00586-005-1046-8.

17. Carr AJ, Gibson B, Robinson PG. Measuring quality of life: is quality of life determined by expectations or experience? BMJ 2001;322:1240-3. 10.1136/bmj.322.7296.1240.

Page $12 / 14$ 
18. Centers for Disease Control and Prevention Measuring healthy days. Atlanta, Georgia, November, 2000.

19. Costenbader EC, Zule WA, Coomes CM. The impact of illicit drug use and harmful drinking on quality of life among injection drug users at high risk for hepatitis C infection. Drug Alcohol Depend. 2007 Jul 10;89(2):251-8.

20. Laudet AB. The Case for Considering Quality of Life in Addiction Research and Clinical Practice. Addict Sci Clin Pract. 2011 Jul;6(1):44-55.

21. Health related quality of life and its correlates among the elderly in Cameroon: Case of Biyem-Assi and Mbengwi Health districts I Cameroon Health Research Forum [Internet]. [cited 2021 Jan 27]. Available from: http://cahref.masante-cam.org/node/219

22. Andong AM, Ngouadjeu EDT, Bekolo CE, Verla VS, Nebongo D, Mboue-Djieka Y, et al. Chronic complications and quality of life of patients living with sickle cell disease and receiving care in three hospitals in Cameroon: a cross-sectional study. BMC Hematol. 2017 Apr $20 ; 17(1): 7$.

23. Kouotou EA, Nansseu J, Tuekam E, Tatah S, Sieleunou I, Claude E, et al. Atopic Dermatitis in Cameroon: Quality of Life and Psychiatric Comorbidities among Affected Children and Adolescents Running Head: Atopic Dermatitis and Psychiatric Impairments. Clin Pediatr Dermatol. 2017 Jan 1;03.

24. Kouotou EA, Adegbidi H, Bene Belembe R, Sieleunou I, Nansseu JR, Kamga J-P, et al. [Acne in Cameroon: Quality of life and psychiatric comorbidities]. Ann Dermatol Venereol. 2016 Oct;143(10):601-6.

25. Communes et villes unies du Cameroun, bureau national, Mamfe. [Internet]. [cited 2021 Aug 9]. Available from: http://www.cvucuccc.com/national/index.php/en/about-uccc/the-secretariat?id=414

26. Population and Housing Census Of Cameroon, 2015 - Cameroon Data Portal [Internet]. [cited 2021 Aug 9]. Available from: https://cameroon.opendataforafrica.org/PHCC2015/population-and-housing-census-of-cameroon-2015?region=1000150-mamfe

27. RAOSOFT. Sample Size Calculator 2020. Available online at: http://www.raosoft.com/samplesize.html (accessed 2 March, 2020).

28. Garin N, Zurita B, Velasco C, Feliu A, Gutierrez M, Masip M, et al. Prevalence and clinical impact of recreational drug consumption in people living with HIV on treatment: a cross-sectional study. BMJ Open. 2017 Jan 1;7(1):e014105.

29. Saitz R, Cheng DM, Allensworth-Davies D, et al. The ability of single screening questions for unhealthy alcohol and other drug use to identify substance dependence in primary care. J Stud Alcohol Drugs 2014;75:153-.

30. Alcohol units. nhs.uk. 2018. https://www.nhs.uk/live-well/alcohol-support/calculating-alcohol-units/. Accessed 14 Jun 2021.

31. WHO | What is Moderate-intensity and Vigorous-intensity Physical Activity? WHO.

https://www.who.int/dietphysicalactivity/physical_activity_intensity/en/. Accessed 7 Apr 2021.

32. WHOQOL - Measuring Quality of Lifel The World Health Organization [Internet]. [cited 2021 Jan 18]. Available from: https://www.who.int/tools/whoqol

33. Hand C. Measuring health-related quality of life in adults with chronic conditions in primary care settings. Can Fam Physician 2016;62:e375-83.

34. Skevington SM, McCrate FM. Expecting a good quality of life in health: assessing people with diverse diseases and conditions using the WHOQOL-BREF. Health Expect 2012;15:49-62. 10.1111/j.1369-7625.2010.00650.x.

35. Veeri RB, Gupta AK, Pal B, Siddiqui NA, Priya D, Das P, et al. Assessment of quality of life using WHOQOL-BREF in patients with visceral leishmaniasis. Health Qual Life Outcomes. 2019 Mar 28;17(1):53.

36. Moreira T de C, Figueiró LR, Fernandes S, Justo FM, Dias IR, Barros HMT, et al. Quality of life of users of psychoactive substances, relatives, and non-users assessed using the WHOQOL-BREF. Ciênc Amp Saúde Coletiva. 2013 Jul;18(7):1953-62.

37. Moreira T de C, Figueiró a R, Maristela Ferigol, Fernandes S, Larré MC, Barros HMT. Quality of Life of Smokers Seeking Help to Quit Through Telephone Counseling. 
38. Campêlo SR, Barbosa MA, Dias DR, Caixeta CC, Leles CR, Porto CC. Association between severity of illicit drug dependence and quality of life in a psychosocial care center in BRAZIL: cross-sectional study. Health Qual Life Outcomes. 2017 Dec;15(1):1-10.

39. Life in War Zones Remains Grim, with Cities Turned into Death Traps, Civilian Suffering 'Pushed to the Limits', Secretary-General Tells Security Council | Meetings Coverage and Press Releases [Internet]. [cited 2021 Aug 15]. Available from: https://www.un.org/press/en/2017/sc12841.doc.htm

40. Domingo-Salvany A, Brugal MT, Barrio G, González-Saiz F, Bravo MJ, La Fuente L, ITINERE Investigators. Gender differences in health related quality of life of young heroin users. Health Qual Life Outcomes 2010; 8:145. 